\title{
Refractive Indices of Thallium Bromide-Iodide Crystals for Visible and Infrared Radiant Energy ${ }^{1}$ -
}

\author{
By Leroy W. Tilton, Earle K. Plyler, and Robert E. Stephens
}

\begin{abstract}
The refractive indices of two specimens of thallium bromide-iodide $\left(\mathrm{KRS}_{5}\right)$ were measured at a constant room temperature of $27^{\circ} \mathrm{C}$ from 0.577 to 39 microns by use of a minimumdeviation spectrometer and a Perkin-Elmer infrared spectrometer equipped with a calibrated screw for operation of the Littrow mirror. In order to cover the entire region over which the prisms are transparent, many known wavelengths were used, including lines of mercury, sodium, hydrogen, and helium for the visible region. A cadmium-mercury lamp was the source from 1 to 2 microns. From 2 to $39 \mu$ various absorption bands of carbondioxide, water, methylcyclohexane, polystyrene, and polyethylene were used, with a Globar as source, for the identification of wavelengths.
\end{abstract}

Mixed crystalline thallium bromide-iodide, a material known as $\mathrm{KRS}-5$, has a very high refractive index, 2.63 for sodium light, and also a high density. It is opaque to wavelengths shorter than about $0.5 \mu$ but very transparent in the infrared to about $40 \mu$. The material is a solid solution of thallium bromide and thallium iodide. That is, it is truly a mixed crystal of the cubic system and therefore not double refracting when free from strain. Interferometer photographs indicate that some of the material is produced in a reasonably homogeneous state, although certainly not as uniform as good optical glass.

For growing these crystals, commercially obtainable chemicals of reagent quality are further purified by crystallization, and then proper mixtures are melted and well stirred. The Germans report that a crystal nucleus is introduced at the top and that the whole is cooled from the upper surface, but it is also successfully grown from the bottom in this country. Crystalline KRS-5 was of great importance to the Germans in the design of optical systems transmitting infrared energy. Incidentally, thallium bromide and iodide have been more or less known to petrographers for 15 or $20 \mathrm{yr}$ as powders that could be mixed and

1 The work described in this paper was carried out in 1947 in part under contract S \& A 28658 between the Bureau of Ships and the National Bureau of Standards, in order to confirm and extend our knowledge of the optical properties of this medium. melted for producing a series of solid immersion media having sodium-lines indices ranging from about 2.40 to 2.65 accordingly as the proportion of thallium iodide is varied from 0 to 75 percent. Solid solutions with more than 75 percent of the iodide component are not stable in the cubic system but invert into a birefringent orthorhombic modification. ${ }^{2}$

Crystals of KRS- 5 have been grown by a number of investigators in the United States and are now grown by the Harshaw Chemical Co. of Cleveland. The measurements of refractive index herein reported were made on a $25^{\circ}$ prism with faces about 6 by $6.2 \mathrm{~cm}$, from a crystal grown by Harshaw, and on a $12^{\circ}$ prism with faces about 4 by $6.5 \mathrm{~cm}$, from a crystal grown by Francis P. Phelps at this Bureau. These prisms were made in the Bureau's Optical Shop, and the surfaces were flat within a few wavelengths over about 80 percent of their apertures. Both these prisms were designed to contain approximately 58 percent of thallium iodide. The Phelps prism was analyzed by Howard B. Knowles of this Bureau and found to contain 58.3 percent of thallium iodide

\footnotetext{
2 Tom. Barth, Am. Mineral. 14, 358 (1929). The curves of refractive index for the visible region versus composition by weight, figure 2 of Barth's paper, seem too low in the neighborhood of $60 \%$ thallium iodide by about 0.03 in index, as judged by the data of this paper on the Phelps prism which, by analysis, contains $58.3 \%$ thallium iodide. Similarly, Barth's curves would seem too low by 0.05 if judged by the Hettner and Leisegang data on a prism said to contain $56 \%$ thallium iodide. (See footnote 4 .)
} 
and 41.7 percent of thallium bromide. The Harshaw prism has not been analysed, because it is in almost constant use on a long program of investigations in the infrared. Moreover, since this material is very susceptible to deformation by stress or shock, it seems unwise to jeopardize the homogeneity of this uniquely valuable optical component by the mechanical operations necessary in removing a sample for analysis. Later it is hoped that an analysis can be made in order to confirm the reasonable inference that this prism is about 0.5 percent higher in thallium iodide content than is the Phelps prism, which has refractive indices only 0.002 lower than those of the Harshaw prism.

The refractive indices of these prisms were first measured in the visible region for eight wavelengths in the yellow and red. This was done by use of a minimum-deviation spectrometer, chiefly at room temperatures near $23^{\circ} \mathrm{C}$. The results for each prism were expressed by a KettelerHelmholtz dispersion formula of the form

$$
n^{2}=a^{2}-k \lambda^{2}+\frac{m}{\lambda^{2}-\lambda_{v}^{2}},
$$

in which the parameters $a^{2}, k, m$, and $\lambda_{v}^{2}$ were adjusted by the method of averages. The average residual of $7 \times 10^{-5}$, somewhat larger than anticipated, may be ascribed in part to somewhat imperfect surfaces and some inhomogeneity, but also it is due in large part to lack of careful temperature control and observation for these preliminary data with visible energy. Also, since this material is practically opaque to visible radiation shorter than about $5,000 \mathrm{~A}$, the indices measured in the yellow region are affected to some extent by the fact that the yellow light is more predominantly transmitted near the apex and perhaps not at all through the thicker portions of the prism near its base. Therefore, the effective apertures for yellow and for red light do not exactly coincide. The resulting error in index measurement is probably appreciable in the yellow region but of little importance in the red and beyond.

Subsequent measurements for $\lambda=5,876$ and $\lambda=6,678$ at $30^{\circ} \mathrm{C}$ were much lower than those near $23^{\circ}$, and a temperature coefficient of $-16 \times 10^{-5} / \mathrm{deg} \mathrm{C}$ was found for light of approximately 6,000 A. This optical sensitivity to temperature is over four times that of rock salt.
The method to be described for the index meas. urements in the infrared is not self-sufficient. For each prism the adjusted index (computed by eq 1 ) for the cadmium red line, $\lambda=6,438$, is taken as the basis with respect to which the infrared indices are to be computed. The use of eq 1 for adjusting the data in the visible region probably compensates in large part for the inadequate knowledge of temperatures of the KRS-5 during individual observations. These basic indices for cadmium red at an average temperature of $23^{\circ}$ were then reduced to $27^{\circ} \mathrm{C}$, the temperature of the room in which the infrared observations were made.

In the infrared region use was made of a PerkinElmer infrared spectrometer equipped with a calibrated screw for operation of the Littrow mirror, $M$ of figure 1 . The concave collimating mirror, $C$, focal length $27.5 \mathrm{~cm}$, approximately $18^{\circ}$ off axis, has an aperture of about $7.5 \mathrm{~cm}$ but was stopped down to a rectangular aperture of about $5 \times 5 \mathrm{~cm}$ to reduce stray energy. The angular separation of the entrance and exit slits is $1.9636^{\circ}$ at the face of the Littrow mirror. The angular equivalent of the screw was found to be $27.05^{\prime}$ or $1623^{\prime \prime}$ per turn, averaged over almost its total length. This was done by means of 64 successive steps with two mirrors that were set at an angle of $5^{\prime}$ of arc. A collimator and telescope were used for precision in making the steps. Evidence was found that seemed to indicate that the periodic errors of a single turn may be $\pm 10 \mathrm{sec}$, and that progressive corrections to the average equivalent of the screw may sometimes exceed 30 sec, but it is possible that this was merely evidence of distortion of the Littrow mirror, introduced by clamping.

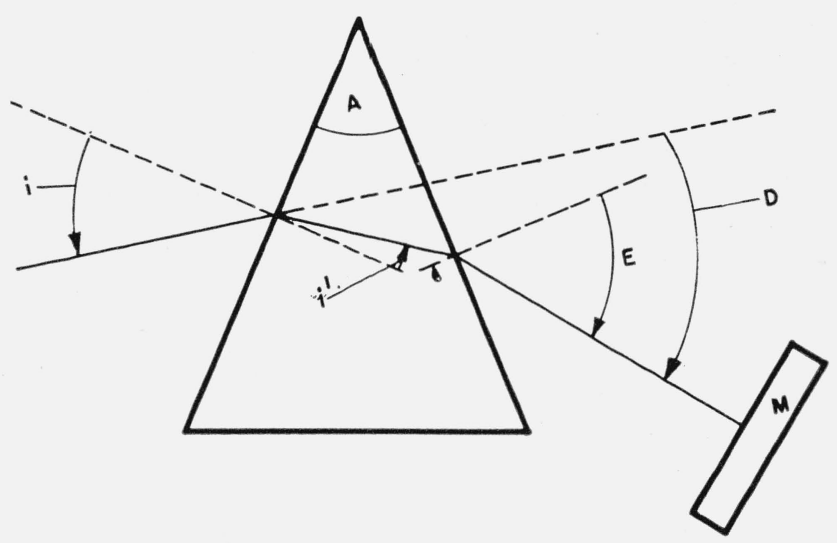

Figure 1. Prism installed on infrared spectrometer. 
The detector was of the Golay type with a KRS-5 window, the incident beam being interrupted about 10 times per sec. Slit widths as wide as 0.35 to $0.43 \mathrm{~mm}$ were necessary for visible radiation and for the region where the water vapor absorption was utilized. Widths of from 0.03 to $0.20-\mathrm{mm}$ were sufficient in the intermediate region. A measure of the energy received by the detector is automatically recorded on a Leeds \& Northrup Speedomax recording potentiometer.

This spectrometer was used, not because it is particularly favorable for this work, but because it was available and index values were required. An objection is the return passage of energy through the prism, which causes additional losses by reflection and absorption. This is particularly disadvantageous in that the energy does not exactly retrace its path because of the angular separation of entrance and exit slits.

Since on the infrared spectrometer the prism is stationary, and only the rotation of the Littrow mirror may be observed, it is necessary to use the method of known incidence angle for refractiveindex measurements with a spectrometer of this type. This method of known incidence was used by Rydberg ${ }^{3}$ over a century ago, and Martens ${ }^{4}$ found it useful in measuring indices in the ultraviolet. Martens recommended the computation of indices by the equations

$\tan \left(i^{\prime}-\frac{A}{2}\right)=\tan \frac{A}{2} \cot \frac{A+D}{2} \tan \left(i-\frac{A+D}{2}\right)$,

and

$$
n=\frac{\sin i}{\sin i^{\prime}}
$$

especially when logarithms are used. For use with computing machines it is preferable to use the equivalent forms

$$
\cot i^{\prime}=\cot A+\frac{\sin E}{\sin A \sin i},
$$

and

$$
n=\sin i\left(1+\cot ^{2} i^{\prime}\right)^{1 / 2},
$$

where the symbols have the meanings designated in figure 2 .

\footnotetext{
3 F. Rydberg, Poggendorf's Annalen 14, 45 (1828).

${ }^{4}$ F. F. Martens, Verhandl. deut. physik. Ges. 3, 10 (1901).
}

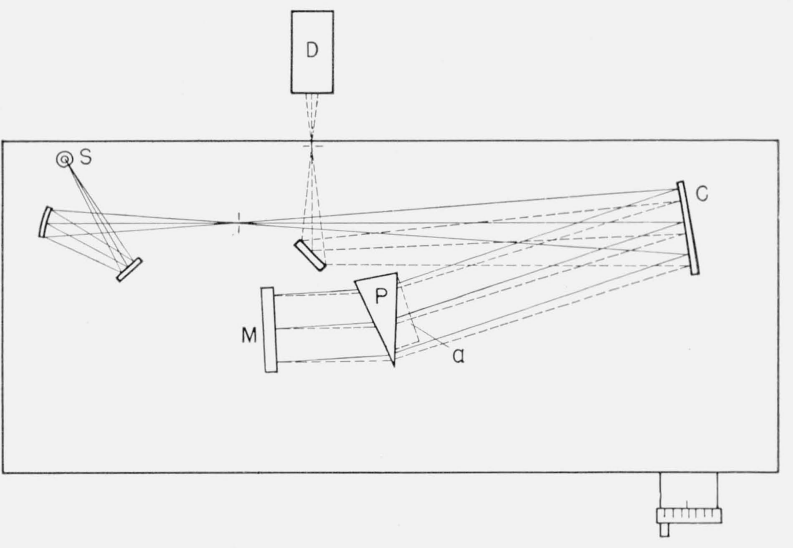

Figure 2. Simplified relation of Littrow mirror to deviation produced by prism.

In order to install each prism of $\mathrm{KRS}-5$ with suitable known incidence angle, $i$, on an infrared spectrometer of this type, small auxiliary prisms of glass were made with angle, $A_{x}$, approximately equal, in each case, to $(\mathrm{A}+\mathrm{D}) / 2$ for the minimumdeviation condition and some wavelength of visible radiant energy traversing the KRS-5 prism. Although there is nothing very precise about the selection of a favorable incidence angle, $i$, it is necessary to know the $i$ that is actually used. Consequently, the angles of the auxiliary glass prisms are carefully measured, and they are used in the installation of the corresponding prisms of KRS-5 on the spectrometer as shown at $A$ in figure 1 . With any intense visible radiation focused on the entrance slit, the prism pair is so oriented that light reflected from the outer polished face of the auxiliary prism is returned to the entrance slit. After removal of the auxiliary prism, any energy from the entrance slit will be incident on the unknown sample prism at an angle $i=A_{x}$, where $A_{x}$ is the measured refracting angle of the auxiliary glass prism.

For any prism, starting with the refracting angle, $A$, the index, $n$, as adjusted for $\lambda_{0}=6,438, t=27^{\circ}$, and incidental angle, $i$, it is possible to trace rays through the prism, as indicated in figure 2, and find $E_{0}$, angle of emergence for these basic or initial conditions. If the Littrow mirror were normal to the emergent ray (zero angle between entrance and emergence slits), or even if the incidence angle of $\delta E / 2$ on the Littrow mirror remained constant for the different wavelengths to be used, then the observable angular increments for the Littrow mirror, as determined from the 
drum readings of the screw, would be the exact increments applicable to $E_{0}$ to give $\mathrm{E}_{\lambda}$.

Fortunately, $\delta E / 2$, the incidence angle on the Littrow mirror, varies rather slowly as $n$ changes, and it is not difficult to trace a series of pairs of rays (for $i$ and for $i-1.9636^{\circ}$ ) for arbitrarily selected evenly spaced values of $n$, as $2.60,2.50$, $2.40,2.30$, and form an interpolation table of $\delta E / 2$ as a function of $n$. Then when computing $n$ by means of eq 1 , it is found that correct preestimation of $n$ within 1 or $2 \times 10^{-3}$ will enable one to select a satisfactory value for the small correction to the observed angular increment in $E$, for use in computing $n$ correctly within \pm 1 or $2 \times 10^{-5}$.

In order to cover the entire infrared region over which these prisms are transparent, many wavelengths were used, including those for various absorption bands of carbondioxide, water, methylcyclohexane, polystyrene, and polyethylene, with a Globar as source. The ordinarily sharp zero branches of the absorption curves for various vapors could not be used, because the recorded contours of these branches are not sharp at the center when the dispersion is low as is the case for these prisms from 3.4 to $14.3 \mu$. Here, however, the recordings of the ordinarily broad bands of absorption of polystyrene, polyethylene, and methylcyclohexane become usefully narrow.

Equation 1 was found definitely inadequate tor accurately representing the index of refraction, even to four decimal places, over so long a spectral range as that covered in this investigation. Also, the four-constant Sellmeier formula with two terms, namely,

$$
n^{2}=1+\frac{M^{\prime} \lambda^{2}}{\lambda^{2}-\lambda_{r}^{2}}+\frac{m^{\prime} \lambda^{2}}{\lambda^{2}-\lambda_{v}^{2}}
$$

exactly equivalent to one form of the KettlerHelmholtz formula (as strictly limited to two resonance frequencies), namely,

$$
n^{2}=a^{2}-\frac{M}{\lambda_{r}^{2}-\lambda^{2}}+\frac{m}{\lambda^{2}-\lambda_{r}^{2}},
$$

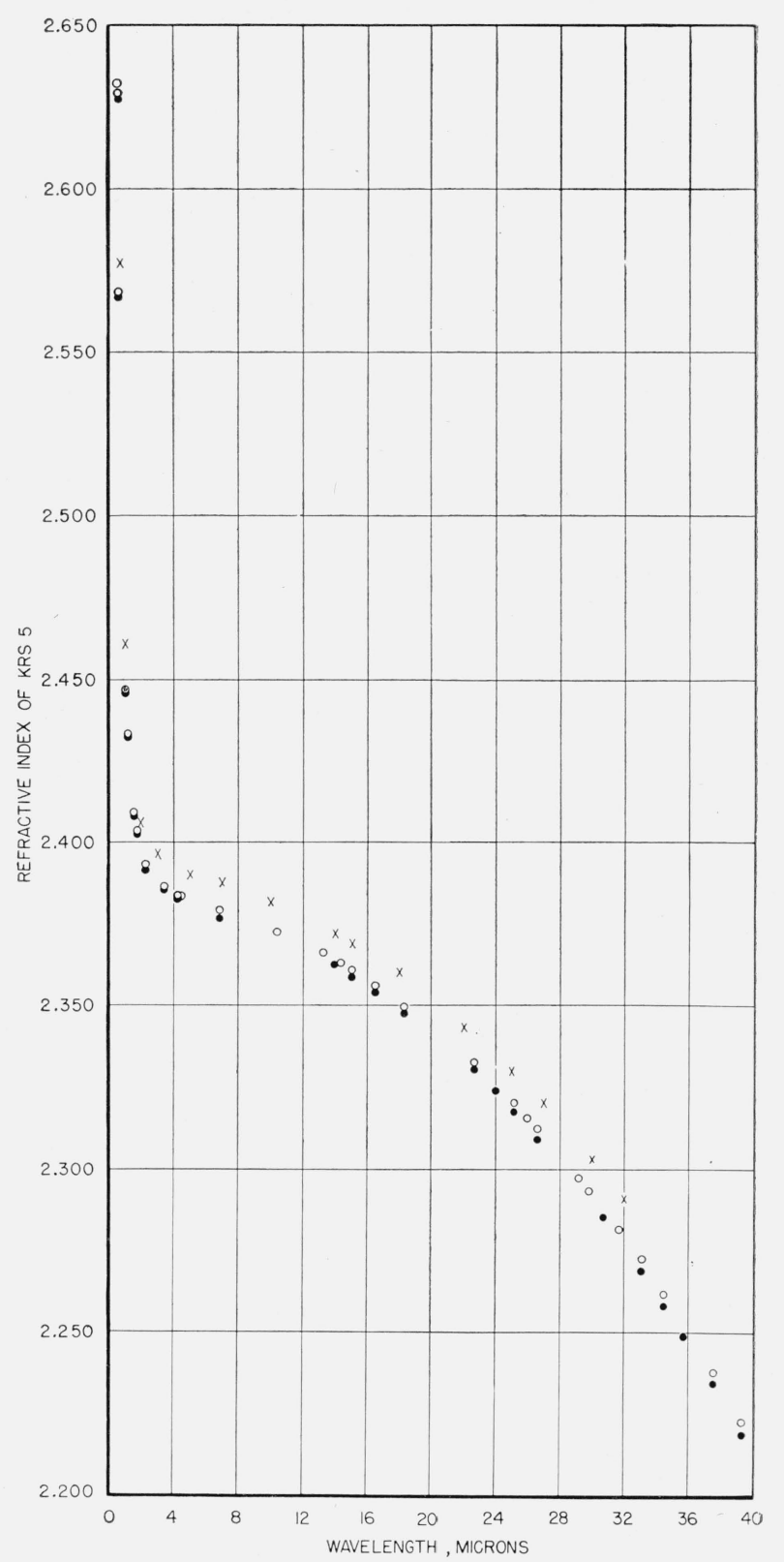

Figure 3. Index of refraction of KRS-5.

The raw observed values for the Harshaw and the Phelps prisms are used here, this graph being identical with that shown at the New York meeting of the Optical Society of America, March 1948. 
where

$$
a^{2}=1+\frac{M}{\lambda_{r}^{2}}+\frac{m}{\lambda_{v}^{2}}=1+M^{\prime}+m^{\prime},
$$

was found to be considerably worse than eq 1 , which is merely a partially expanded, and therefore an approximate, form of eq 4 or 5 . Equation 4 or 5 can, however, account for the effect of only one absorption band in the infrared and one in the ultraviolet, whereas their expanded forms can, with the same number of constants, approximate the effects of multiple bands, and these prisms of KRS-5 are known to have two prominent infrared bands, one each for the bromide and iodide constituents. By retaining in the expansion a term in $-\lambda^{4}$, however, and adjusting the five parameters by least squares, the equation

$$
\begin{gathered}
n^{2}=5.676927-0.0004522264 \lambda^{2}- \\
0.0000000180197 \lambda^{4}+ \\
\frac{0.2873017}{\lambda^{2}-0.1027734},
\end{gathered}
$$

was found to represent the indices of the $25^{\circ}$ Harshaw prism.

The same equation with $a^{2}=5.667927$ serves so well (see residuals in table 1 ) for the $12^{\circ}$ Phelps prism that a betterment by least squares is unnecessary.

Equation 5 with five constants, that is one in which

$$
a^{2}=1+\frac{M}{\lambda_{r}^{2}}+\frac{m}{\lambda_{v}^{2}}+\ldots .
$$

was not tried because if five constants are to be used, it appears much better to try the expanded form as in eq 6 , where not only the constant, but certain other additional terms relating to any other pertinent absorption bands are automatically taken into account.

Table 1 gives, according to eq 6 and 7 , the indices and the residuals, observed minus computed, for each of these prisms for the wavelengths that were used. The agreement between observed and computed indices for each prism is entirely satisfactory, and the computed indices for these prisms are probably typical for this material.

\begin{tabular}{|c|c|c|c|c|c|}
\hline $\begin{array}{l}\text { Wave- } \\
\text { length }\end{array}$ & $\begin{array}{c}\text { Source or absorbing } \\
\text { media }\end{array}$ & $\begin{array}{l}\text { Com- } \\
\text { puted } \\
\text { index } \\
\text { Har- } \\
\text { shaw } \\
\text { prism }\end{array}$ & $(0=c) \times 10^{4}$ & $\begin{array}{l}\text { Com- } \\
\text { puted } \\
\text { index } \\
\text { Phelps } \\
\text { prism }\end{array}$ & $(0=c) \times 10^{4}$ \\
\hline$\mu$ & & & & & \\
\hline 0.5770 & Hg yellow & 2. 6316 & +1 & & \\
\hline .5780 & Mean $\mathrm{Hg}$ yellow .... & & & 2. 6286 & -14 \\
\hline .5791 & Hg yellow ..... & 2. 6291 & +1 & & \\
\hline .6438 & Cd red.... & 2. 5687 & -6 & 2. 5670 & -6 \\
\hline 1. 0142 & $\mathrm{Hg} \ldots$ & 2. 4468 & +3 & 2.4450 & +13 \\
\hline 1. 1289 & $\mathrm{Hg}_{-.}$ & 2. 4334 & +2 & 2.4316 & +11 \\
\hline 1. 5298 & $\mathrm{Hg} \ldots$ & 2. 4092 & +3 & 2. 4073 & +12 \\
\hline 1. 700 & $\mathrm{Hg}_{2} \ldots$ & 2. 4039 & +2 & 2. 4020 & +11 \\
\hline 2. 26 & Polyethylene abs.... & 2. 3942 & -5 & 2. 3923 & -5 \\
\hline 3.42 & Polystyrene abs & 2. 3867 & +2 & 2. 3848 & +10 \\
\hline 4. 24 & $\mathrm{CO}_{2}$ abs. in air. & 2. 3843 & -1 & 2. 3824 & +8 \\
\hline 4. 4 & $\mathrm{CO}_{2}$ flame emission... & 2. 3839 & -4 & .... & ….. \\
\hline 6. 86 & $\begin{array}{l}\text { Methyleyclohexane } \\
\text { abs. }\end{array}$ & 2. 3794 & +1 & 2.3775 & -4 \\
\hline 10. 34 & do & 2. 3730 & +0 & -... & (n....... \\
\hline 13. 23 & Folystyrene abs..... & 2. 3662 & +3 & -..- & .... \\
\hline 13. 90 & Polyethylene abs... & & & 2.3625 & +6 \\
\hline 14.32 & Polystyrene abs .... & 2. 3632 & +1 & .... & 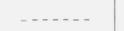 \\
\hline 14. 98 & $\mathrm{CO}_{2}$ abs. in air......... & 2. 3613 & -1 & 2.3594 & -2 \\
\hline 16. 48 & $\begin{array}{l}\text { Methylcyclohexane } \\
\text { abs. }\end{array}$ & 2. 3567 & -2 & 2.3547 & -1 \\
\hline 18. 29 & ..... do do ...... & 2. 3504 & -4 & 2.3485 & -3 \\
\hline 22. 60 & _. do do & 2. 3328 & +1 & 2. 3308 & +3 \\
\hline 23. 92 & Water vapor abs. ${ }^{\mathrm{a}}$ & 2. 3265 & +3 & 2.3246 & +1 \\
\hline 25. 16 & … do.a ${ }^{a} \ldots$ & 2. 3203 & +0 & 2.3184 & -4 \\
\hline 25.97 & -... do. ${ }^{a} \ldots$ & 2,3160 & -1 & ... & -.... \\
\hline 26. 60 & -...do. do & 2. 3127 & -3 & 2.3107 & -7 \\
\hline 28.33 & ... do. ${ }^{\mathrm{a}}$ & $\cdots$ & 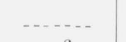 & 2. 3008 & -2 \\
\hline 29. 15 & .... do. ${ }^{\mathrm{a}}$ & 2. 2978 & -6 & -... & …... \\
\hline 29. 81 & -.... do. ${ }^{\mathrm{a}} \ldots$ & 2. 2937 & -1 & 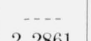 & +2 \\
\hline $\begin{array}{l}30.69 \\
31.70\end{array}$ & $\begin{array}{c}\text { do. }{ }^{\mathrm{a}} \ldots \\
\ldots \text { do. }{ }^{\mathrm{a}} \ldots\end{array}$ & 2. 2814 & -0 & $\begin{array}{c}2.2861 \\
\ldots\end{array}$ & +2 \\
\hline 33. 11 & do ${ }^{\mathrm{a}}$ & 2. 2715 & +10 & 2. 2695 & 0 \\
\hline 34.48 & do. ${ }^{a} \ldots$ & 2. 2614 & +1 & 2. 2594 & -7 \\
\hline 35.7 & $\ldots$ do. ${ }^{a} \ldots$ & ... & $\ldots$ & 2. 2500 & -7 \\
\hline 37.45 & $\ldots$ do $a_{\ldots} \ldots$ & 2. 2377 & -3 & 2. 2357 & -6 \\
\hline 39. 22 & do. ${ }^{a}$ & 2. 2224 & -1 & 2. 2204 & -6 \\
\hline
\end{tabular}
The differences in index from wavelength to wavelength, easily obtainable from table 2 , are probably accurate to a few units in the fifth decimal. The
TABLE 1. Observed and computed data on index or refraction of $\mathrm{KRS}-5\left(27^{\circ} \mathrm{C}\right)$

a For the wavelengths of the absorption bands of water vapor, see H. M. Randall, D. M. Dennison, N. Ginsburg, and L. R. Weber, Phys Rev. 52, 160 (1937)

index residuals for the Phelps prism are larger, because the effects on index of accidental errors in deviation measurement are fully twice as large for the prism of $12^{\circ}$ angle. Moreover, since the resolution is much lower, the angular errors themselves are larger. The average difference in raw observed index between the two prisms varies from $13 \times 10^{-4}$ in the visible region to $28 \times 10^{-4}$ at $39 \mu$, figure 3 , whereas the corresponding differences are 17 to $20 \times 10^{-4}$ in the computed indices. In other 
This table is an average for two samples of KRS-5 grown independently in different laboratories in the United States in efforts to produce solid solutions of minimum melting point. One, by analysis, contains 58.3 percent thallium iodide and the other, by inference from its indices, contains 58.8 percent thallium iodide. The measurements were made at 35 wavelengths well distributed over the range of $40 \mu$. The table is computed by means of equation 6 , except that $a^{2}$ has the value 5.672427. It is estimated that differences taken from this table are reliable to $\pm 2 \times 10^{-5}$.

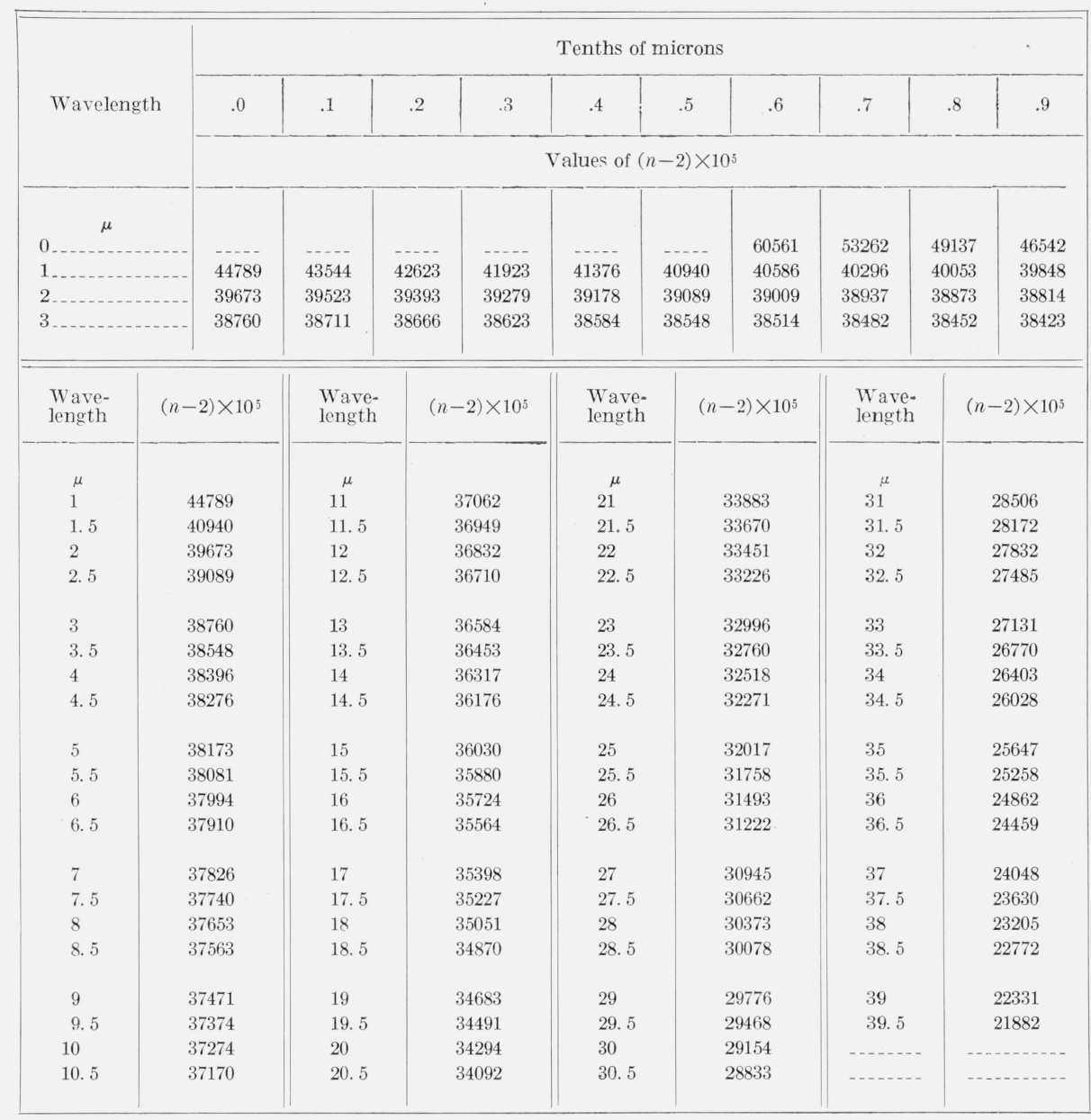

words, there is little if any difference in dispersion between these two prisms. The corresponding difference, if any, in the proportions in which the bromide and the iodide are present would be about 0.5 percent, the Harshaw prism to have the slightly higher iodide content.

Figure 3 shows by open circles the (observed) indices of the $25^{\circ}$ prism grown by Harshaw and by black dots the indices of the $12^{\circ}$ prism grown by Phelps. Some indices of a sample of KRS-5 according to "Hettner", ${ }^{5}$ as read from a curve ob-

\footnotetext{
${ }^{5}$ Possibly based on the data pubiished later by G. Hettner and Gertrud Leisegang, Optik 3, 305 (1948).
}

tained from Germany, are entered on this figure as crosses. They extend, however, only to $32 \mu$. At the long wavelengths that sample of KRS-5 averaged fully $100 \times 10^{-4}$ higher in index than the Phelps prism even allowing for a possible difference of $10 \mathrm{deg}$ C. This indicates, according to the index curves published by Barth, that the Hettner sample contained about 2.5 percent more thallium iodide than the Phelps prism. ${ }^{6}$

${ }^{6}$ The Hettner and Leisegang prism (footnote 4) is said, however, to contain $56 \%$ of thallium iodide, which is about $2.3 \%$ less than that in the Phelps prism. With reference to index stability, it may be added that check measurements for $\lambda=6438$ were made on the Phelps prism after a 15 -month interval, using a different spectrometer, and no change was found in the fourth decimal place. 